\title{
MCMARTHY, Martha, From the Great River to the Ends of the \\ Earth: Oblate Missions to the Dene, 1847-1921 (Edmonton, University of Alberta Press/Western Canadian Publishers, 1995), xxiii-269 p.
}

\section{Claude Champagne}

Volume 51, numéro 1, été 1997

URI : https://id.erudit.org/iderudit/305631ar

DOI : https://doi.org/10.7202/305631ar

Aller au sommaire du numéro

Éditeur(s)

Institut d'histoire de l'Amérique française

ISSN

0035-2357 (imprimé)

1492-1383 (numérique)

Découvrir la revue

Citer ce compte rendu

Champagne, C. (1997). Compte rendu de [McMARTHY, Martha, From the Great River to the Ends of the Earth: Oblate Missions to the Dene, 1847-1921

(Edmonton, University of Alberta Press/Western Canadian Publishers, 1995), xxiii-269 p.] Revue d'histoire de l'Amérique française, 51(1), 112-115.

https://doi.org/10.7202/305631ar d'utilisation que vous pouvez consulter en ligne. 
McMARTHY, Martha, From the Great River to the Ends of the Earth: Oblate Missions to the Dene, 1847-1921 (Edmonton, University of Alberta Press/Western Canadian Publishers, 1995), xxiii-269 p.

Martha McMarthy nous offre une histoire de l'activité missionnaire des oblats auprès des Dene, de l'arrivée du père Alexandre Taché dans l'Atha- 
baska jusqu'à la signature du traité avec les Dene du Mackenzie en 1921. L'auteure se signale en se proposant de donner les deux versions de cette histoire: celle des missionnaires oblats, assez bien documentée et connue des historiens, mais également celle des «missionnés», qui s'en tiennent à la tradition orale. Pour ce faire, elle a séjourné dans le Mackenzie, interviewant plusieurs anciens (elders) parmi les Dene.

Les deux premiers chapitres situent les deux principaux acteurs de cette mission: le monde «ancien», que représentent les oblats, et le monde «nouveau», celui des différents groupes Dene avec leur diversité. Les oblats eurent à se situer par rapport à deux autres groupes présents dans le même territoire: la Compagnie de la Baie d'Hudson, responsable politique du territoire jusqu'en 1859 (chap. 3) et les missionnaires anglicans de la Church Missionary Society (chap. 4). L'auteure offre un jugement nuancé sur ces différents groupes. Le chapitre 5 rappelle que la mission catholique de l'époque cherchait à fournir un clergé et à établir des structures diocésaines comme partout dans le monde catholique-romain.

Le chapitre 6 décrit la rencontre entre ces deux mondes: les oblats et les Dene. L'auteure soutient qu'eut lieu un vrai dialogue entre les deux partenaires, malgré leurs grandes différences. Les oblats visaient la conversion des Dene au catholicisme sans être éveillés à la dimension culturelle. Elle souligne les différents éléments de la tradition religieuse dene et les méthodes d'approche des oblats, méthodes déjà utilisées en France. Les principaux problèmes soulevés par cette rencontre oblat-dene se situaient autour des questions de mariage et de la polygamie.

Suit un intéressant chapitre (7) sur le leadership parmi les Dene. En l'absence du prêtre, des leaders chrétiens ont émergé. L'auteure présente quelques-unes des grandes figures de leaders dene, dont Clémence Thanizeneaze, et mentionne la prédication publique par une femme dans une communauté catholique dene, réalité inconcevable à l'époque dans l'Europe catholique. Ces leaders dene ont proclamé l'Évangile en tenant compte du contexte et de la culture dene.

Le chapitre 8 souligne le rôle important des métis, nés de pères canadiens-français et de mère cri ou chipewyan. Certains ont servi de modèles aux Dene. Leur familiarité avec le catholicisme et la langue française ont favorisé le travail missionnaire des oblats, au détriment de la Church Missionary Society.

Le chapitre 9 traite de la santé et du bien-être, et de la place de la médecine dans l'activité missionnaire des oblats. Tant pour les Dene que pour les oblats, il existe un lien entre la santé et la foi, mais l'auteure va trop loin en affirmant que les soins de santé étaient alors une partie intégrante de l'évangélisation (p. 119). Bien sûr que les oblats ont vu un devoir religieux, une œuvre de bienfaisance dans l'engagement pour soulager les malades et les sauver de la mort. Mais de là à reconnaître dans cette activité une partie intégrante de la mission comme on le ferait maintenant, j'hésite à l'affirmer. L'auteure décrit l'introduction de maladies européennes avec l'arrivée des 
Blancs; or, les Dene, tout comme les autres autochtones d'Amérique du Nord, n'avaient pas développé les anticorps nécessaires pour résister à ces maladies. C'est dire que l'histoire missionnaire est marquée par une série d'épidémies. Certains leaders dene ont réagi aux épidémies en accusant les missionnaires d'en être la cause (chap. 10). L'auteure fait l'intéressant historique de cette résistance à l'action des oblats.

Le chapitre suivant (11) touche le rapport entre l'éducation et l'évangélisation dans l'œuvre missionnaire des oblats. La Church Missionary Society et les oblats voyaient dans l'éducation un outil d'évangélisation pour gagner les cœurs et les esprits des Dene. Les missionnaires catholiques percevaient cet engagement en vue de la civilisation chrétienne. L'auteure mentionne la place de la langue dene dans ces écoles et la réalité des orphelinats où les enfants devaient travailler afin de soutenir l'institution. Les oblats ne voyaient pas dans la vie nomade de pêcheur et de chasseur un obstacle à l'Évangile et à la vie chrétienne, mais les écoles ont rendu les élèves inaptes à la vie nomade, tout en ne les préparant pas de manière suffisante à la vie des Blancs. De plus, l'auteure, sensible à la problématique contemporaine, souligne avec raison la douleur de la séparation des jeunes enfants et de leurs parents. Le soutien financier des écoles fut toujours problématique: l'œEuvre de la Sainte-Enfance assura le financement jusqu'à ce que le gouvernement, avec le traité 8 de 1899, accepte une responsabilité de soutenir les écoles. Mais, pour ce dernier, il demeurait plus économique de confier les écoles aux différentes Églises.

Après les traités, le gouvernement mena une politique d'acculturation agressive et d'assimilation où seules les langues anglaise et française étaient acceptées. Cette politique eut un effet dévastateur, conduisant à l'oubli de la langue dene. On niait ainsi la culture dene. Si certains élèves ont apprécié leur expérience de l'école, d'autres en ont souffert, parlant d'abus et de système qui leur était étranger. L'auteure, quittant la période 1847-1921, rappelle la demande de pardon des oblats en juillet 1991, au Lac-Sainte-Anne (Alberta). Les oblats percevaient les aspects négatifs des écoles résidentielles, mais sans pouvoir imaginer une alternative.

Les oblats furent des intermédiaires entre les Dene et le gouvernement fédéral (chap. 12). Ils ont également cherché à améliorer la situation de leurs missions. C'est ainsi qu'ils ont été associés à la négociation du traité 8 de 1899 et du traité 11 de 1921 . S'ils y ont vu des avantages dans le domaine de l'éducation - financement des écoles par le gouvernement -, ils ont protesté avec les Dene et les Métis contre les intrusions du gouvernement dans la vie des gens. L'auteure souligne que la signature des traités a créé de la distance entre les oblats et les Dene.

Le dernier chapitre fait fonction de conclusion. Les Dene ont manifesté leur ouverture aux nouvelles formes de puissance spirituelle. Certains ont embrassé la foi catholique sans nécessairement accepter toutes les règles peu conformes à leur style de vie, comme l'assistance à la messe dominicale. D'autres ont rejeté la foi catholique au profit de leur religion traditonnelle ou 
de la foi anglicane. Si certains ont été des catholiques fervents, d'autres ont vécu un dualisme religieux entre catholicisme et religion traditionnelle. D'autres, enfin, ont eu une approche plus syncrétique dans laquelle le cadre de référence a pu être ou le catholicisme ou la religion traditionnelle.

L'ouvrage se termine par des appendices sur les statistiques de la population dene, sur les maladies, sur les chefs spirituels, de même que sur les missions oblates auprès des Dene. Quelques cartes géographiques accompagnent le texte, mais elles ne sont pas très claires et aident peu le lecteur.

Somme toute, un ouvrage bien fait qui donne voix aux Dene, tout en reconnaissant l'apport positif des oblats dans l'œuvre missionnaire. 